\title{
Humoral immunity to tetanus, measles and rubella in children with acute lymphoblastic leukemia after chemotherapy
}

\author{
Myriam Onoratelli, M.D. ${ }^{a}$, Claudia Botana, M.D. ${ }^{a}$, Laura Peralta, M.D. ${ }^{a}$, Magalí Rebollo, M.D. ${ }^{a}$, \\ Silvina Ruvinsky, M.D. ${ }^{a}$, Myriam Guitter, M.D. ${ }^{a}$, María S. Felice, M.D. ${ }^{a}$, Mercedes Posadas, M.D. ${ }^{a}$, \\ Silvina Evangelista, M.D. ${ }^{a}$, María V. Villar, M.D. ${ }^{a}$, Mariana Golluscio, M.D. ${ }^{a}$, Agustina Molina, M.D. ${ }^{a}$, and \\ Lidia Fraquelli, M.D. ${ }^{a}$
}

\begin{abstract}
Chemotherapy regimens and clinical support advances have improved survival in children with acute lymphoblastic leukemia. The after-effects of treatment are a reason for concern, including damage to the immune system induced by immunosuppressive therapy which is reflected in the loss of antibody protection provided by prior immunizations.

Our goal was to assess the presence of measles, rubella, and tetanus protective antibody titers among patients with acute lymphoblastic leukemia after completing chemotherapy.

Sixty-one children with acute lymphoblastic leukemia seen at the Hospital Garrahan were included; patients had finished their chemotherapy at least 6 months earlier and had a complete immunization schedule before diagnosis. The rates of protective antibodies were $46 \%$ (CI: 32-59) for measles, 53\% (CI 40-67) for tetanus, and 60\% (CI 47-63) for rubella.

These results strengthen the need to reconsider revaccination in this group of patients.

Key words: acute lymphoblastic leukemia, immunizations, humoral immunity, antibodies, chemotherapy.
\end{abstract}

http:/ / dx.doi.org/10.5546/aap.2016.eng.549

\section{INTRODUCTION}

Over the past two decades, intensified chemotherapy regimens and advances in clinical support implementation have improved survival among children with acute lymphoblastic leukemia (ALL). ${ }^{1}$

The increasing number of survivors poses a challenge for pediatricians, especially in relation to the development of sequelae secondary to treatment. These include damage to the immune system induced by immunosuppressive therapy. Children with ALL who are treated with chemotherapy suffer from humoral and

a. Hospital de Pediatría SAMIC Prof. Dr. Juan P.
Garrahan, Autonomous City of Buenos Aires.

E-mail address: Myriam Onoratelli, M.D.: onoratelli@yahoo.com.ar

Funding: None.

Conflict of interest: None.

Received: 12-13-2015

Accepted: 7-28-2016 cellular immunosuppression, which may last for months, or even years, after they have finished treatment. $^{2-4}$

Immunizations are highly important for the prevention of communicable infectious diseases. In this context, it is relevant to know the level of protection against vaccine-preventable diseases in these patients, who received immunizations in a timely manner and then underwent chemotherapy.

There is little evidence in the literature regarding the recovery of antibody protective levels provided by prior immunizations in children treated for ALL who completed their chemotherapy. Published studies report different results, so it is not possible to make a definite recommendation on a revaccination policy. ${ }^{5}$ In our setting, no data have been published in this regard.

The objective of this study was to assess the presence of measles, rubella, and tetanus antibody protective titers among patients with ALL after chemotherapy.

\section{POPULATION AND METHODS}

We conducted a descriptive, observational, cross-sectional study.

Sixty-one patients diagnosed with ALL and seen at the Hospital de Pediatría Prof. Dr. Juan P. Garrahan between June 2008 and January 2013 were included. Inclusion criteria were having received first-line treatment as per the ongoing protocol, being 1 to 17 years old at the time of diagnosis, having completed chemotherapy 6 to 18 months before the study, and having received every vaccine indicated in the national immunization schedule.

Patients included in the study agreed to participate in a voluntary and informed manner and gave their consent and assent, if applicable.

Treatment corresponded to Protocol 11-ALLIC/BFM-2002. ${ }^{6}$ Patients were classified into three risk groups (standard, intermediate and high) as per clinical, biological and treatment response parameters. Treatment intensity differed across risk groups. 
Compliance with immunizations was confirmed by checking the patients' vaccination card.

Children who had received the corresponding booster doses of tetanus, measles or rubella vaccine for their age after chemotherapy, patients with immune disorders prior to ALL diagnosis, and those who missed the corresponding booster doses for their age because they were receiving chemotherapy were excluded.

The main outcome measures were rubella, measles and tetanus antibody titers.

Antibody titers were measured 6 to 18 months after chemotherapy completion; for this reason, and given that no measurement was done prior to chemotherapy, the situation was defined as "absence" of protective levels instead of "loss." Although these results were not compared to antibody levels prior to chemotherapy, it was considered that, in the general population who have completed their immunization schedule, the prevalence of antibody protective titers against the studied diseases ranged between $90 \%$ and $95 \% .^{7-10}$

Immunoglobulin $G(\operatorname{Ig} G)$ titers for rubella were determined using a quantitative method based on the microparticle enzyme immunoassay (MEIA) from Abbott ${ }^{\circledR}$ Laboratories.

IgG titers for measles were determined using a qualitative method which associated the twostep sandwich enzyme immunoassay to the enzyme linked fluorescent assay (ELFA) from bioMérieux®.

Antibody titers against the tetanus toxoid were determined at the immunology laboratory using the enzyme-linked immunosorbent assay (ELISA).

In addition, for each case, the child's age at the time of diagnosis, time (in months) elapsed after chemotherapy, and ALL risk group were recorded.

In all cases, a single determination was made from a blood sample drawn from a vein, which was requested together with the routine cancer control of these patients. No additional blood draws were made for the purpose of this study.

The study was approved by the Research Review Committee and the Ethics Committee of the hospital.

\section{Statistical analysis}

For the descriptive analysis of continuous outcome measures, mean, median and standard deviation were used as summary statistics based on data distribution. Categorical outcome measures were established as absolute and percent values.

Protective rates were expressed as percentage and $95 \%$ confidence interval (CI) to report on the accuracy of the value obtained.

The relationship among the presence or absence of protective titers and ALL risk groups, age at the time of diagnosis, and time elapsed after chemotherapy was also analyzed. The STATA 9.0 (StataCorp USA) statistical software was used for analysis.

\section{RESULTS}

Sixty-one children were included in the study: 31 girls and 30 boys. Table 1 describes the main characteristics of the population.

The rate of protective antibodies was $46 \%$ (95\% CI: 32-59) for measles, 53\% (95\% CI: 40-67) for tetanus, and $60 \%$ (95\% CI: 47-63) for rubella.

The presence of protective antibodies by risk group was as follows:

- Measles: $53 \%$ in patients who had a standard risk, $50 \%$ in those who had an intermediate risk, and none in the six patients who had a high risk.

- Rubella: 53\% in patients who had a standard risk, $65 \%$ in those who had an intermediate risk, and $50 \%$ in those who had a high risk.

- Tetanus: $53 \%$ in patients who had a standard risk, $57 \%$ in those who had an intermediate risk, and 33\% in those who had a high risk.

The descriptive analysis of our results did not show a difference in the presence of protective antibodies and median age at the time of diagnosis or median time (in months) elapsed after chemotherapy completion (Tables 2 and 3).

TABLE 1. General characteristics of the studied population $(n=61)$

\begin{tabular}{lc}
\hline Male sex, $\mathrm{n}(\%)$ & $30(49)$ \\
$\begin{array}{l}\text { Age (years old) at the time of antibody } \\
\text { titer measurement; median (range) }\end{array}$ & $10.3(3.6-19.1)$ \\
\hline ALL risk group, $\mathbf{n}(\%)$ & $16(26)$ \\
$\quad \begin{array}{l}\text { Standard } \\
\text { Intermediate }\end{array}$ & $39(64)$ \\
$\begin{array}{l}\text { High } \\
\text { Time (months) elapsed after } \\
\text { chemotherapy; median (range) }\end{array}$ & $6(10)$ \\
\hline
\end{tabular}

ALL: acute lymphoblastic leukemia. 


\section{DISCUSSION}

In our study, it was observed that, depending on the antibody assessed, between $40 \%$ and $54 \%$ of children diagnosed with ALL who completed conventional chemotherapy lacked protective antibodies against measles, rubella and tetanus, although they had received these vaccines in accordance with the national immunization schedule before they started chemotherapy.

Children with ALL who are treated with chemotherapy suffer from humoral and cellular immunosuppression, which may last for months or even years after they have finished treatment. ${ }^{2-4}$ Humoral immunity expressed by B cell function may be assessed measuring immunoglobulin serum levels. It has been observed that, at the end of chemotherapy, these levels are close to the $10^{\text {th }}$ percentile and reach normal values within six months after completing immunosuppressive therapy. ${ }^{2,4}$

However, there is little evidence in the literature regarding the recovery of antibody protective levels provided by immunizations received prior to chemotherapy.

Nilsson et al. ${ }^{11}$ analyzed antibody titers in 43 children after chemotherapy and demonstrated the persistence of protection against measles and rubella in $60 \%$ and $72 \%$ of patients, respectively. Brodtman et al. ${ }^{12}$ studied antibody titers provided by several vaccines in 100 children with ALL and observed that the percentage of these children who had protective titers was remarkably lower than that expected for immunized control subjects. In the study conducted by Von der Hardt et al., ${ }^{13}$ more than $50 \%$ of patients lacked

TABLE 2. Presence of protective titers against measles, rubella and tetanus by age at the time of diagnosis $(n=61)$

\begin{tabular}{|c|c|c|c|}
\hline \multirow[t]{2}{*}{$\begin{array}{l}\text { Antibody } \\
\text { protective titers }\end{array}$} & & \multicolumn{2}{|c|}{$\begin{array}{c}\text { Age at } \\
\text { diagnosis of ALL }\end{array}$} \\
\hline & & X SD & $(95 \% \mathrm{CI})$ \\
\hline \multirow[t]{2}{*}{ Measles antibodies } & + & $6.9 \pm 4.5$ & $(5.1-8.6)$ \\
\hline & - & $6.9 \pm 4.2$ & $(5.5-8.4)$ \\
\hline \multirow[t]{2}{*}{ Tetanus antibodies } & + & $7.2 \pm 3.8$ & $(5.9-8.6)$ \\
\hline & - & $6.7 \pm 5$ & $(4.9-8.5)$ \\
\hline \multirow[t]{2}{*}{ Rubella antibodies } & + & $7.2 \pm 4.3$ & $(5.7-8.6)$ \\
\hline & - & $6.7 \pm 4.4$ & $(4.9-8.4)$ \\
\hline
\end{tabular}

ALL: acute lymphoblastic leukemia.

X SD: median and standard deviation. CI: confidence interval.

+ Presence of antibody protective titers.

- Absence of antibody protective titers. protective immunity to diphtheria and tetanus after chemotherapy.

Published studies describe different results and, although many show reduced protective antibody titers, some are under scrutiny due to their design.

Only one systematic review has been done, by Van Tilburg et al., ${ }^{5}$ and its results were described as protective rate ranges, which seemed adequate given study variety. The wide range of protective antibody titers among studies, which may be explained by their heterogeneity and limited sample size, prevented the chance of doing a meta-analysis. So it was not possible to make a definite recommendation on a revaccination policy. ${ }^{5}$

The United Kingdom and Spain recommend the administration of a booster dose of all vaccines received six months after completing chemotherapy. These recommendations are mainly based on expert opinions and a limited number of published studies. ${ }^{14,15}$

Some studies have demonstrated that the incidence of reduced protective antibody titers is higher among younger patients. ${ }^{11}$

The descriptive analysis of our results did not show a difference between the presence of protective antibodies and median age at the time of diagnosis or the median time (in months) elapsed after chemotherapy completion.

Although the percentage of protective antibodies has been described for the different ALL risk groups, one of the limitations of these results is that the high risk group included only six patients.

TABLE 3. Presence of protective titers against measles, rubella and tetanus by months elapsed after chemotherapy $(n=61)$

\begin{tabular}{lccc}
\hline Antibody protective titers & & \multicolumn{2}{c}{$\begin{array}{c}\text { Months elapsed } \\
\text { after chemotherapy }\end{array}$} \\
& & X SD & (95\% CI) \\
\hline Measles antibodies & + & $8.9 \pm 2.5$ & $(7.9-9.9)$ \\
& - & $9.2 \pm 2.5$ & $(8.4-10.1)$ \\
Tetanus antibodies & + & $8.7 \pm 2.2$ & $(7.9-9.5)$ \\
& - & $9.4 \pm 2.7$ & $(8.4-10.4)$ \\
Rubella antibodies & + & $9.5 \pm 2.6$ & $(8.6-10.4)$ \\
& - & $8.6 \pm 2.3$ & $(7.6-9.5)$ \\
\hline
\end{tabular}

XSD: median and standard deviation. CI: confidence interval. + Presence of antibody protective titers.

- Absence of antibody protective titers. 
Therefore, although none of the high risk patients were observed to have protective antibodies against measles, the small sample size prevents us from detecting a significant difference.

It is worth noting that even patients with a standard risk did not evidence a high percentage of protective antibodies.

Therefore, the results of our study strengthen the need to reconsider revaccination policies for patients who receive chemotherapy for ALL, including those who are in the standard or intermediate risk group and receive a less intense chemotherapy regimen, and especially those who have a high risk and receive an intensified regimen.

\section{Acknowledgments}

We would like to thank Susana Patricia Rodríguez, M.D., ${ }^{*}$ and Stella Carchio, M.D. ${ }^{* *}$ for their help to prepare and produce this article.

* Associated Director of Research and Teaching of Hospital de Pediatría SAMIC "Prof. Dr. Juan P. Garrahan".

** Head of Laboratory.

\section{REFERENCES}

1. Smith MA, Ries LA. Childhood cancer: incidence, survival, and mortality. In: Pizzo PA, Poplack DG, eds. Principles and practice of pediatric oncology. 4th ed. Philadelphia: Lippincott-Williams and Wilkins; 2002.Págs.1-12.

2. Alanko S, Pelliniemi TT, Salmi TT. Recovery of blood B-lymphocytes and serum immunoglobulins after chemotherapy for childhood acutelymphoblastic leukemia. Cancer 1992;69(6):1481-6.

3. Alanko S, Salmi TT, Pelliniemi TT. Recovery of blood T-cell subsets after chemotherapy for childhood acute lymphoblastic leukemia. Pediatr Hematol Oncol 1994;11(3):281-92.
4. MustafaMM, Buchanan GR, WinickNJ,McCracken GH, etal. Immune recovery in children with malignancy after cessation of chemotherapy. J Pediatr Hematol Oncol 1998;20(5):451-7.

5. Van Tilburg CM, Sanders EA, Rovers MM, Wolfs TF, et al. Loss of antibodies and response to (re-)vaccination in children after treatment for acute lymphocytic leukemia: a systematic review. Leukemia 2006;20(10):1717-22.

6. Stary J, Zimmermann M, Campbell M, Castillo L, et al. Intensive chemotherapy for childhood acute lymphoblastic leukemia: results of the randomized intercontinental trial ALL IC-BFM 2002. J Clin Oncol 2014;32(3):174-84.

7. Centro Nacional de Epidemiología. Instituto de Salud Carlos III. Estudio seroepidemiológico: situación de las enfermedades vacunables en España. Madrid. Ministerio de Sanidad y Consumo; 2000.

8. Pachón I, Amela C, De Ory F. Age specific seroprevalence of poliomyelitis, diphtheria and tetanus antibodies in Spain. Epidemiol Infect 2002;129(3):535-41.

9. Pedranti MS, Adamo MP, Macedo R, Zapata MT. Prevalencia de anticuerpos antirrubéola y antiparvovirus B19 en embarazadas de la ciudad de Córdoba y en mujeres en edad fértil de la ciudad de Villa Mercedes, San Luis. Rev Argent Microbiol 2007;39(1):47-50.

10. Pichichero ME, Anderson EL, Rennels MB, Edwards KM, et al. Fifth vaccination with diphtheria, tetanus and acellular pertussis is beneficial in four-to six-year-olds. Pediatr Infect Dis J 2001;20(4):427-33.

11. Nilsson A, DeMilito A, EngströmP, Nordin M, etal.Current chemotherapy protocols for childhood acutelymphoblastic leukemiainduceloss of humoralimmunity toviralvaccination antigens. Pediatrics 2002;109(6):e91.

12. Brodtman DH, Rosenthal DW, Redner A, Lanzkowsky $\mathrm{P}$, et al. Immunodeficiency in children with acute lymphoblasticleukemiaaftercompletionofmodernaggressive chemotherapeutic regimens. J Pediatr 2005;146(5):654-61.

13. Von der Hardt K, Jüngert J, Beck JD, Heininger U. Humoral immunity against diphtheria, tetanus and poliomyelitis after antineoplastic therapy in children and adolescents-a retrospective analysis. Vaccine 2000;18(26):2999-3004.

14. Royal College of Paediatrics and Child Health (RCPCH). Immunization of the immunocompromised child: Best Practice Statement. United Kingdom: RCPCH; 2002.

15. Mellado Peña MJ, Moreno-Pérez D, Ruíz Contreras J, Hernández- Sampelayo Matos, et al. Documento de consenso de la Sociedad Española de Insectología Pediátrica y el Comité Asesor de Vacunas de la Asociación Española de Pediatría para la vacunación en inmunodeprimidos. An Pediatr (Barc) 2011;75(6):413.e1-22. 